stands the idea has a seductive and perhaps illusory simplicity. If its basic thesis is correct then it may in fact be even more difficult to unravel the circuitry and causal relationships involved in membrane signal transduction processes than was earlier supposed.

1 Cuatrecasas, P. in Specificity and Action of Animal, Bacterial and Plant Toxins (ed. Cuatrecasas, P,) Receptors and Recognition (Series B) (Chapman

Perkins, J. P. in Adv. Cyclic Nucleotide Res. 3, 2

ef kowitz, R. J. Limbird, L. E. Mukherjee, C. \& aron, M. G. Biochim. biophys. Acta 457, I (1975).

Bennett, V. O'Keefe, E. \& Cuatrecasas, P. Proc. natn. Acad. Sci. U.S.A. 72, 33 (1976)

Helmrich, E. J. M Zenner, H. P Pfeuffer, T \& Cori $\mathrm{C} . \mathrm{F}$ in Current Topics in Cellular Regulation C. F. in Current Topics in Cellular Regulation (1976)

${ }^{4}$ Rodbell, M. in Current Topics in Biochemistry, 187 (Academic, New York, 1972)

Sonnenberg, M. \& Schreider, A. S. in Receptors and Recognition (eds Cuatrecasas, P. \& Greaves, M. F.) Series A, 3 (Chapman and Hall, London, in the press).

* Cuatrecasas, P. A. Rev. Biochem, 43, 169 (1974).

Beers, R. F. \& Bassett, E. G. (eds) Cell Membrane Receptors for Viruses, Antigens and Antibodies, Polypeptide Hormones, and Simall Molecule Raven, New York, 1976).

10 Cohen, J.'P. \& Changeux, J. -P. A. Rev. Pharmacol. $15,83(1975)$.

Greaves, M. F. in Receptors and Recognition (eds. Cutrecasas, P. \& Greaves, M. F.) Series A, 1, 1 (Chapman and Hall, London, 1975)

12 Lemay, A. \& Jarett, L.J. Cell Biol. 65, 39 (1975).

${ }^{3}$ Rendell, M. Salomon, Y. Lin, M. C. Rodbell, M. \&

Berman, M. J. biol. Chem. 250, 4253 (1975)

Bennett, V. Rec. Progr. Hormone Res, Ki, 37 Bennett, is Jacobs, $\dot{\mathrm{S}}$. \& Cuatrecasas, P. Biochim. biophys. Acta
$\mathbf{4 3 3}, 482$ (1976).

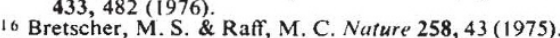

17 McGuire, R. F. \& Barber, R. J. supramolec. Structure 4, 259 (1976)

18 Jarrett, L. \& Smith, R. M. in Cell Membrane Receptors for Viruses, Antigens and Antibodies, Polypeptide Hormones, and Small Molecules (eds Beers, R. F. \& Bassett, E. G.), 91 (Raven, New York, 1976).

19 Bretscher, M. S. Nature 260, 21 (1976).

20 Orly, J. \& Schramm, M. Proc. natn. Acad. Sci. U.S.A. 73, 4410 (1976).

21 Birnbaumer, L. \& Rodbell, M. J. biol. Chem. 244 3477, (1969).

22 Coffino. P. et al. 32, 669 (1976).

\section{Breakdown of superfluidity in liquid helium}

from A. J. Leggett

IT has been known for more than 60 years that liquid helium $\left({ }^{4} \mathrm{He}\right)$ is superfluid below about $2 \mathrm{~K}$; that is, it flows through narrow capillaries without observable friction, and, conversely, an object moving slowly through ${ }^{4} \mathrm{He}$ at low temperatures experiences practically no friction either. A major step forward in understanding this phenomenon was made by the Russian physicist Lev Landau in 1941; he showed that superfluidity is a natural consequence of certain assumptions about the "elementary excitations" of the liquid, and predicted the critical velocity above which normal behaviour should set in. In a recent paper (Phil. Trans. R. Soc. 284, 179; 1977) D. R. Allum et al. report the first unambiguous observation of this 'Landau critical velocity', thereby strongly confirming Landau's ideas. They also report some surprising and significant features of the mechanism by which superfluidity breaks down above the critical velocity.

An 'elementary excitation' is the simplest (quantised) disturbance which can be created in a given system; generally, it will carry a momentum $p$ and an energy $E$ which depends on $p$. Landau argued that at sufficiently low temperatures a heavy external body travelling through the system can experience friction only if it can create elementary excitations. However, conservation of momentum and energy prevent this happening at velocities less than the Landau critical velocity $v_{\mathrm{L}}$ given by

$$
v_{\mathrm{L}}=\min (E(p) / p)
$$

A slight modification of the argument gives the same critical velocity for helium flowing through a capillary. In the case of a normal classical liquid it is easy to show that $v_{\mathrm{L}}$ is zero, so there is no superfluidity.

For liquid helium below $2 \mathrm{~K}$, however, it is believed that the elementary excitation spectrum (the graph of $E(p)$ against $p$ ) should have the form shown in the figure. The excitations of small $p$ (long wavelength) are quantised sound waves, or phonons, whereas those near the minimum of the curve are called 'rotons'. Originally a roton was envisaged as a quantised rotational motion of the liquid, but more recent theories have suggested some modifications to this picture and its physical nature is still somewhat obscure. The existence of rotons and more generally the correctness of the $E(p)$ curve of the figure has been confirmed both by specific-heat data and by experiments in which a neutron is scattered from the liquid by creating a single elementary excitation.

It is obvious from the figure that the minimum of $E(p) / p$ occurs near the roton minimum in the curve and is in fact given by the slope of the straight line; numerically, this corresponds to a value of $v_{\mathrm{L}}$ of about $50 \mathrm{~m} \mathrm{~s}^{-1}$ At velocities higher than this emission of one or more rotons will occur, giving rise to friction. However, experiments on the flow of helium through narrow capillaries usually yield much lower (and often geometry-dependent and/or irreproducible) critical velocities, probably owing to the existence of tangles of vorticity in the liquid.

Allum and coworkers used the alternative technique of measuring the frictional drag on bodies moving through the liquid-in this case, negative ions. (A negative ion in liquid helium is believed to be a complex object composed of an electron plus the cavity it excavates for itself in the liquid and the portion of the helium which moves with it; the whole has a diameter of the order of $10 \AA$ and a

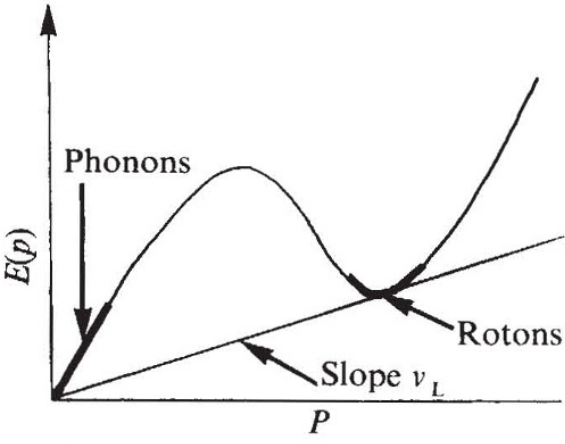

Fig. 1 Elementary excitation spectrum of superfluid helium.

mass of about 70 helium-atom masses). When plotted as a function of electric field $E$, the mean velocity of the ions, $\bar{v}$, rises virtually instantaneously from zero to a value which is of order $50 \mathrm{~m} \mathrm{~s}^{-1}$ and coincides within the experimental error with the value of $v_{\mathrm{L}}$ predicted from the excitation spectrum. This indicates strongly that at velocities less than $v_{\mathrm{L}}$ there is indeed no frictional drag, in accordance with Landau's ideas.

A second interesting and quite unexpected result emerges from a comparison of the observed behaviour of $\bar{v}$ as a function of $E$ with a recent theory, due to R. M. Bowley and F. W. Sheard, of the frictional drag at velocities above $v_{\mathrm{L}}$. They showed that if the dominant process by which the ion loses energy and momentum is creation of single rotons, the quantity $v-v_{\mathrm{L}}$ should be proportional to $E^{2 / 3}$, whereas if it is creation of pairs of rotons which dominates it should be proportional to $E^{1 / 3}$. For most of the range of fields studied the data fit an $E^{1 / 3}$ dependent; they cannot possibly be reconciled with the $E^{2 / 3}$ one. Thus, it seems that negative ions moving in helium create rotons only, or at least predominantly, in pairs whereas neutrons seem to have no difficulty in creating them singly! This intriguing result seems certain to stimulate further research, both experimental and theoretical, into the physical nature of rotons.

\section{Dynamic Stark splitting}

\section{from Peter Knight}

WORKERS in fields other than quantum optics or atomic physics may be surprised to hear that resonance fluorescence, that classic phenomenon of early quantum physics, has shrugged off its antiquarian associations to become a major and intensively studied new research problem. The rejuvenating influence has been the tunable laser. Unlike life, 'undressed' atoms, those old-fashioned atoms bathed in a weak 